\title{
RESEARCH
}

\section{Lesion Segmentation in Breast Ultrasound Images using Optimized Marked Watershed Method}

\author{
Xiaoyan Shen ${ }^{1}, \mathrm{He} \mathrm{Ma}{ }^{1,2^{*}}$, Ruibo Liu ${ }^{1}$, Hong Li ${ }^{1}$ \\ , Jiachuan $\mathrm{He}^{3}$ and Xinran $\mathrm{Wu}^{1}$
}

${ }^{*}$ Correspondence:

mahe@bmie.neu.edu.cn

${ }^{1}$ the College of Medicine and

Biological Information

Engineering, Northeastern

University, Shenyang, China

Full list of author information is

available at the end of the article

\begin{abstract}
Background: Ultrasound is the most popular tool for early detection of breast cancer because of its non radiation and low cost. However, breast ultrasound(BUS) images have low resolution and speckle noise, which make lesion segmentation become a challenge. Most of deep learning(DL) models applied on images segmentation don't have good generalization ability for BUS images.

Therefore, it is time to go back to the classical method and consider combining it with DL to achieve more accurate and efficient effect in a semi-automatic way.
\end{abstract}

Methods: This paper mainly proposed an effective and efficient semi-automatic BUS images segmentation method, Adaptive morphological snake and marked watershed( AMSMW). It includes two parts: preprocessing and segmentation. In the first part, we combine contrast limited adaptive histogram equalization(CLAHE) and side window filtering(SWF) methods for the first time. In the second part, We use the proposed adaptive morphological snake algorithm (AMS) to provide a mark for marked watershed(MW) method.

Results: we tested on 500 BUS images, whose ratio of benign and malignant is 1:1. After quantitative and qualitative analysis, AMSMW is proven to outperform existing classical methods on the effectiveness and efficiency. Furthermore, we compared with Zhuang's RDAU-NET on both our dataset and theirs.

Experimental result showes AMSMW achieved better performance on most of indicators, including loss, accuracy, sensitivity, dice and F1-score.

Conlusions: The new image preprocessing method proposed by us has obvious effect on segmentation of breast ultrasound image. In addition, the proposed adaptive morphology snake method and optimized marked watershed turn out to be more efficient and effective than some relative classical method and the advanced DL method at present. Moreover, by studying on the algorithm's sensitivity in segmenting benign and malignant tumors, we found that AMSMW is more sensitivity to malignant tumors, and more stable to benign tumors, which is significant for further research of precision medicine.

Keywords: Breast; morphology snake; segmentation; side window; Ultrasound; watershed

\section{Background}

According to the 2018 global cancer data report, the incidence rate of breast cancer is $24.2 \%$, and the mortality rate is $15 \%$, indicating that breast cancer has become an important threat to the health of women worldwide [1]. Studies have shown that early detection and diagnosis of breast cancer can effectively improve the cure rate [2]. At present, digital mammography (DM) and breast ultrasound (BUS) are 
widely used in breast disease screening in China. DM is the most commonly used imaging tool in clinical examination. However, However, due to its radiation and sensitivity, DM is not suitable for detecting breast lesions in Asian women with high density. On the contrary, BUS has no radiation and is more sensitive to dense breast, which can increase the overall cancer detection rate by $17 \%$, so BUS is becoming more and more popular [3]. In addition, Geisel et al. also demonstrated the effectiveness, practicability and feasibility of BUS as a screening tool for early detection of occult breast cancer [4]. However, in the process of BUS imaging, the speckle noise generated by coherent waves greatly reduces the image quality, which is very unfavorable for radiologist to make diagnosis.

With the development of artificial intelligence technology, the computer aided diagnosis (CAD) system based on medical images has made great achievements in cancer detection. As the key step of CAD system, medical image segmentation is to segment the diseased tissue region (region of intetrest, ROI) from the normal tissue. Only through accurate medical image segmentation, can we obtain complete pathological tissue information, including shape, texture, density and other features, which can provide an important basis for the identification of benign and malignant tumors. However, due to the inherent characteristics of BUS images, the existence of speckle noise and low resolution, BUS images segmentation has become a challenge.

In recent years, many excellent segmentation algorithms have emerged. Level set (LST), first introduced in 1994 [5], and improved in the year of 1995 [6], 2005 [7], $2012[8]$ and so on, has proven to be very effective in image segmentation. However, it costs a lot of time to solve partial deferential equations (PDEs), which is not very practical. In order to solve this problem, morpholical snake (MS) was proposed. It used morphological operations on a binary level set to approach the differential operators of standard PDE [9]. It just needs numerical calculations, which is much simpler, faster and stable. In addition, it doesn't require re-initialization because the level set is just a binary piecewise constant function. In terms of the field of BUS images segmentation, Many scholars [10], [11], [8] used parameter deformable model and geometric deformable model technology. They should set a good initial tumor boundary or a precise edge based stop function in advance. Some researchers have used and improved the graph based segmentation method [12] and [13]. Boukerroui attempted to overcome the biggest drawbacks of MRF model, i.e., lower optimization speed and local optimization [14]. In 2013, Zhao proposed generalized fuzzy cluster method (FCM) with spatial information, which performs well in segmentation and have a rapid converge speed [15]. FCM and improved FCM algorithm were applied on lesion detection in BUS images [16], [17]. Since 2013, there have been more and more segmentation methods with supervised and semi supervised learning, especially the popularity of deep learning (DL), which has made great progress in solving the problem of BUS images segmentation. The supervised learning includes support vector machine (SVM), artificial neural network (ANN), Convolutional neural network (CNN), etc., which were applied to BUS images segmentation and made great progress ([18], [19], [20], [21]) and Zhuang proposed RDAU-NET model [22], which behaves best on segmenting lesions when compared to other DL modes. So far, DL has been proven to be the best way to perform image segmentation. However, DL faces some major problems: the prediction result 
is not robust enough, model can't be explainable and data is not enough, which are also the main bottleneck for the further development of DL. In order to solve this, a new approach was proposed to integrating visual saliency into a deep learning model for breast tumor segmentation in ultrasound images [23]. They introduced attention blocks into a U-Net architecture and learns feature representations that prioritize spatial regions with high saliency levels, and achieved a Dice similarity coefficient (DSC) of $90.5 \%$ on a data set of 510 images. However, this method relies much on the quality of saliency maps, which is also not robust enough. Therefore, it is time to go back to the classical methods and consider to combine DL with excellent traditional methods. Many excellent classical segmentation methods are worth learning from. Tomography watershed (WS) has a certain effect on solving complex segmentation problems and is more stable compared to other existing methods, but it is sensitive to noise and might cause over-segmentation. In view of this, many scholars have improved WS. Huang and Chen [10] combined WS with the active contour model to obtain a relatively accurate tumor boundary. In 2009, Gomez used marked watershed (MW) algorithm incorporating morphological techniques and ARD function [24]. They improved the method by using anisotropic diffusion filter (ADF) guided by texture descriptors derived from a set of Gabor filter and creating segmenting function generated by Newton filters to facilitate more precise segmentation [25]. However, ADF needs many iterations to get a good preprocessing result, which takes a long time. In addition, the acquisition of marker function is a little complex, which will reduce the efficiency of the algorithm. In view of these two problems, we have made improvements in previous study [26]. We combined contrast limited adaptive histogram equalization (CLAHE) and curvature filtering $(\mathrm{CF})$ to preprocess images, and used morphological method to obtain the marker function, which is simple and efficient. However, this method not only improves the segmentation accuracy and Dice coefficient, but also brings higher FPR value, which means that many false positive tissues are also segmented. Therefore, to further improve the performance, this paper makes several technical contributions, which are concluded into the following four tips.

1. For the first time, the side window filter (SWF) was used to filter the BUS images processed by CLAHE to enhance the edge of lesion tissue. Compared with other preprocessing methods, it is found that the preprocessing method used in this paper is the most beneficial to BUS images segmentation.

2. We proposed adaptive morphological snake (AMS), which is more robust and stable when processing complex data set with different sizes of lesions collected from different types of ultrasound equipment, compared with morphology snake (MS).

3. We proposed an optimized marked watershed segmentation method, Adaptive morphological snake marked watershed(AMSMW). Its' marker is corrected by AMS. Taking full consideration of the advantages of classical segmentation algorithm, such as level set (LST) method [5], morphological snake(MS) [9] and MW [27], we found that AMSMW has higher segmentation precision and also 3-4 times faster than other existing methods.

4. Quantitative and qualitative analysis were presented comprehensively and objectively with traditional segmentation methods and DL models based methods. 


\section{Results}

$C L A H E+S W F$ is the best preprocessing method.

Quantitative result and some examples of qualitative result are shown in the Table 3 and Figure 5 separately. Firstly, as shown in the Table 3, no indicators in the first column is better, indicating that the preprocessing method is vital and has a very important effect on the result of BUS image segmentation. Secondly, although the CLAHE+SWF method is lower than SWF on the TPR value, it behaves best on the other indicators. This indicates CLAHE can improve the contrast and SWF can smooth the noise, preserving lesion boundary greatly, which can also be seen intuitively from Figure 5 .

AMSMW behaves best both on quantitative result and on qualitative result.

Firstly, some relevant and excellent traditional segmentation methods were tested on our testing set, and the quantitative and qualitative results are shown later. It can be seen from the Table 4 that even without the preprocessing method, MW still performs the best on TPR, which indicates that MW can segment the entire lesion area more sensitively and comprehensively, whereas the error rate is also raised to the highest, causing too high FPR. That means a large part that does not belong to the lesion area is also segmented, which can be seen intuitively from the qualitative result in the Figure 6. Take the image in the third and fourth column for example, a lot of normal tissue are segmented by MW. Whereas, LST has a lower FPR compared to MW, indicating that it can effectively improve the ability of identifying lesion boundaries. However, other indicators' values of LST are relatively low, indicating that it cannot completely find entire lesion area. Compared with LST, MS has the biggest advantage that it uses morphological methods to replace the process of solving numerical differential equations, improving the efficiency greatly. Through experimental verification, MS is 3 to 4 times faster than LST. At the same time, it can be seen from the quantitative results that MS is better than LST in most indicators such as ACC, DSC, JI, AER, HE, and MAE, indicating that it can segment tumors more precisely than LST. However, as shown in the Figure 6, take the third and the fourth column for example. there are some tumors with many calcification points inside, which cannot be segmented completely by MS method, increasing the FPR's value. Compared with MS, although AMS is slightly lower than MS on Acc, FPR, AER, HE and MAE, it has obvious advantages on TPR, DSC and JI, showing that AMS is more stable and can get more complete lesions. This is of great significance for obtaining a better maker of AMSMW later. By comparing MSMW with AMSMW, it is easier to see that AMS has great effect on improving the performance. The LSMW algorithm is a method in which the LST method is used instead of AMS as the segmentation function. By comparing the quantitative results of LSMW with MSMW, AMSMW, and FSMW, AMSMW has obvious advantages on other indicators except TPR. In addition, from the Figure 6 , it is easier to find that the qualitative result of AMSMW is much closer to GT. Moreover, AMSMW method runs fastest. From all the above, we conclude that AMSMW has highest efficiency and effectiveness when segments tumors in complex BUS images.

Secondly, we performed segmentation on the testing set shared by [22] and ours, respectively. The quantitative and qualitative results are shown in Table 5, Figure 
7. and Table 6, Figure 8 , respectively. Seen from Table 5, AMSMW is slightly lower than RDAU-NET on SP, PC and M-IOU, while obviously higher on the other five indicators, which include Acc, Loss, DC, Sen and F1. This indicates that AMSMW has good adaptability and can segment lesion area more precise than RDAU-NET, which can also be seen more intuitively from Figure 8. As Figure 8 shows that RDAU-NET can also find lesion area, whereas it segments more normal tissue area, increasing FPR's value. Furthermore, from the results of experiment on our testing set, RDAU-NET does not show strong generalization ability, because it is only better than AMSMW on Sen, and is far worse than AMSMW on the other seven indicators. On the whole, it is obvious that if there is no enough training set and good hardware resources, even the best deep learning model isn't available when it comes to new or more complex data. Thus, there is still a long way for deep learning to go to improve its performance of generalization. In other words, although traditional algorithms cannot be fully automated, the capability of semi-automated is enough to bear doctors' burden, and excellent traditional segmentation algorithms still have good performance when processing complex data.

\section{Evaluation on the sensitivity in segmenting benign and malignant tumors}

As shown in the Table 7, the performance of AMSMW in segmenting benign and malignant tumors is comparable. From the four indicators, TPR, DSC, JI and AER, we can conclude that AMSMW is more sensitive to discriminating malignant tumors. However, due to the strong echo behind lesion and the possible strong inner calcification points, AMSMW has a high FPR when segments malignant tumors, causing that the algorithm performs poorly on the other four indicators, including ACC, FPR, HE and MAE. Therefore,AMSMW is more stable when segments benign tumors.

\section{Discussion}

By exploring the effect of preprocessing methods on segmentation results, we find that the appropriate preprocessing method is conducive to better image segmentation results. For BUS images segmentation with obvious speckle noise, CLAHE + SWF is a better image processing method. By comparing AMSMW with some classic traditional segmentation methods, we found that AMSMW is the most efficient and effective. This is mainly due to the AMS method which can adaptively change the working parameters without tedious calculation of the PED equation as the segmentation function. By comparing AMSMW with the state-of-art DL models, rdau-net and u-net and so on, AMSMW still performed well on most of the indicators. It proves that AMSMW has strong robustness as a semi-automatic segmentation algorithm. While RDAU-NET has the ability of automatic segmentation, it has weak generalization ability. When dealing with complex images with more speckle noise, different shapes and sizes of tumor and from different ultrasonic equipment, RDAU-NET is far less powerful than AMSMW. The main reason is that the labeled training set is limited and the learning ability of the model is limited. However, in present era of DL, which has attracted much attention and is widely sought after, most of the DL models do not have the ideal generalization ability, which leads to the bottleneck of its continuous development. Wheras, the 
traditional segmentation method is stable and efficient, which provides a way to solve the bottleneck problem of DL to a certain extent. Therefore, in the future work, we should not abandon classical segmentation method. Maybe it is a great solution to integrate the efficient and stable traditional segmentation method with DL model to complete images segmentation work, and the results will certainly be greatly improved.

By evaluating the sensitivity of the algorithm in segmenting benign and malignant tumors, we get that AMSMW has high sensitivity for the delineation of malignant tumor boundary, and is relatively stable for benign tumor. Therefore, in the future, in the improvement of the algorithm, we should take the strong echo and characteristics of malignant tumor into account, and set up an adaptive ideal segmentation method.

\section{Conclusions}

In this paper, an efficient and effective semi-automatic BUS images segmentation method were developed and quantitatively evaluated. In the preprocessing part, by combining the latest and best smoothing and noise-deduced method, SWF, with CLAHE, we preserved the boundary in very large degree, which contributes a lot to doing accurate segmentation. And in the segmentation part, we optimized morphology snake method and input it as the segmentation function of optimized marked watershed, which turns out to be more efficient and effective than FSMW and the advanced DL method at present. Moreover, by studying on the algorithm's sensitivity in segmenting benign and malignant tumors, we found that AMSMW is more sensitivity to malignant tumors, and more stable to benign tumors, which is significant for further research of precision medicine.

\section{Methods}

The flowchart of the proposed method is shown in Figure 1. It is mainly consisted with four parts: data acquisition, pre-processing, marker acquisition and final contour acquisition. The specific steps of each part are listed in the frame.

\section{Data Acquisition}

We collected 500 BUS images, which include 250 benign solid cysts and 250 malignant solid cysts. They are captured from different devices, such as GE LOGIQ E9 and PHILIPS EPIQ 5, in the local hospital. The patient information on all images were hidden. An experienced radiologist sketched lesion boundary for each image as the ground-truth (GT).

\section{RROI Acquisition}

RROI(rectangle region of interest)is obtained manually by the following step: firstly, selecting a point as a starting point by left-clicking the mouse, holding down the left mouse button to move diagonally until the end position is found. Here, we define the RROI's four vertices as $w_{1}, w_{2}, h_{1}$, and $h_{2}$, respectively, where $\mathrm{w}$ and $\mathrm{h}$ represent the point is along the tumor's width and height, respectively, and subindices 1 and 2 represent the lower and upper limits of the tumor's width and height, respectively. The geometric center of the RROI, which will be used later, can be defined as

$$
\hat{\mu}=\left(\mu_{w}, \mu_{k}\right)=\left(w_{1}+\frac{w_{2}-w_{1}}{2}, h_{1}+\frac{h_{2}-h_{1}}{2}\right)
$$




\section{Image Pre-processing}

Contrast Enhancement

BUS images are characterized by low-contrast and much noise, which can be improved by applying CLAHE, an optimization method based on adaptive histogram equalization (AHE) by limiting the increase in contrast. It excellently overcomes the problem of over-amplifying noise that AHE algorithm has.

\section{Edge Highlighting}

Local windows, whose center of the window aligned with the pixels being processed, usually causes blurred edges. In order to avoid this, [28] proposed SWF, which can significantly preserve edges. Thus, we introduced SWF to highlight lesions'edges in BUS images. We will give a brief introduction on SWF, more can be found in the paper [28].

As shown in the Figure 2, eight side windows are only defined in a discrete case, where $(\mathrm{x}, \mathrm{y})$ is the coordinates of target pixel $\mathrm{i}, \mathrm{r}$ and $\theta$ are the radius and angle of the window, respectively. $\rho \in\{\mathrm{o}, \mathrm{r}\}, \theta=\mathrm{k} \times \pi / 2, \mathrm{k} \in[0,3]$. Thus, we can get four side windows, $W_{D i}, W_{R i}, W_{U i}$ and $W_{L i}$, by setting $\rho=\mathrm{r}$, which align i with their sides. While $\rho=0$, we have $W_{S W i}, W_{S E i}, W_{N E i}$ and $W_{N W i}$, which align i with their

corners. For each pixel, the process of filtering can be regarded as the process of finding the $I_{a m}$ value, which satisfies:

$$
I_{\mathrm{m}}=\arg \min _{n \in S}\left\|q_{i}-I_{n}\right\|_{2}^{2}
$$

where,

$$
\begin{aligned}
& \mathrm{I}_{n}=\frac{1}{N_{n}} \sum_{j \in w_{i}^{n}} w_{i j} q_{j} \\
& \mathrm{~N}_{n}=\sum_{j \in w_{i}^{n}} w_{i j}, n \in S
\end{aligned}
$$

$W_{i j}$ is the weight of pixel $\mathrm{j}$, which is neighbored with pixel $\mathrm{i}$, based on the filtering kernel $\mathrm{F}, q_{j}$ is the intensities of image $\mathrm{q}$ at location $\mathrm{i}$, and $\mathrm{S}=\mathrm{L}, \mathrm{R}, \mathrm{U}, \mathrm{D}, \mathrm{NW}, \mathrm{NE}$, SW, SE is the set of side window index. The result of filtering by SWF is defined as:

$$
\mathrm{I}_{\mathrm{SWF}}^{\prime}=\arg \min _{\forall I_{i}^{\theta, \rho, r}}\left\|q_{i}-I_{i}^{\prime \theta, \rho, r}\right\|_{2}^{2}
$$

where

$$
\mathrm{I}_{i}^{\prime \theta, \rho, r}=F\left(q_{i}, \theta, \rho, r\right)
$$

$$
I_{i}^{\prime} \theta, \rho, \gamma \text { is the result of eight side windows, when } \rho \in\{\mathrm{o}, \mathrm{r}\}, \theta=\mathrm{k} \times \pi / 2, \mathrm{k} \in[0,3] \text {. }
$$

\section{Constraint Gaussian kernel set}

Similar to the method proposed in the paper [26], We multiply Gaussian functions with the filtered image $I_{C F}$ to obtain the region of interest(ROI). Whereas the 
difference is we used a union of five constrained Gaussian, which have the same variances, in order to make the lesion area more prominent.

$$
\sigma_{w}=\frac{w_{2}-w_{1}}{2}, \sigma_{h}=\frac{h_{2}-h_{1}}{2}
$$

The only difference between the five constrained Gaussian functions is the center position. One is centered at the geometric center of the RROI, and the other four are translated by half of diagonal lengths to the four diagonal directions of the RROI, respectively. Hence, take the Gaussian function which centered at the geometric center of the RROI as an example, its function could be expressed as

$$
G(m, n)=\frac{\exp \left(-\mathbf{1} / \mathbf{2}\left(\frac{(\hat{p}-\hat{\mu})^{2}}{\sigma_{w}^{2}}+\frac{(\hat{p}-\hat{\mu})^{2}}{\sigma_{h}^{2}}\right)\right)}{2 \pi \sqrt{\operatorname{det} s_{\sigma}}}
$$

where $\hat{p}(\mathrm{~m}, \mathrm{n})$ represents the pixel's location, $s_{\alpha}$ is the diagonal covariance matrix. It could be expressed as:

$$
s_{\sigma}=\left(\begin{array}{cc}
\sigma_{w}^{2} & 0 \\
0 & \sigma_{h}^{2}
\end{array}\right)
$$

Referring to (7), We superimpose these five Gaussian functions to obtain their union $G_{T}$, and then multiply it with $I_{C F}$, which was negative firstly.

$$
J(m, n)=G_{T}(m, n) \bullet\left(\mathbf{1}-\frac{I_{C F}(m, n)}{\max _{\hat{p}}\left(I_{C F}(m, n)\right)}\right)
$$

Therefore, a specially highlighted ROI, whose surrounding tissue is greatly darkened, is obtained as shown in Figure 3. Experiments shows that the illuminated ROI obtained by these five Gaussian function sets are more complete than before, which is significant for determining accurate tumor boundaries and performing efficient segmentation.

\section{Opening operation}

The input image of marker function, JmnM, and the input image of segmentation function, JmnN are obtained separately by performing opening operation on J using 9 pixels disk and 15 pixels radius disk, respectively.

\section{Marker Acquisition}

The marker of MW algorithm is composed of marker function and segmentation function, which is also one of the main innovations of this paper.

\section{Marker function}

Similiar to the paper [26], we get the marker function through a series of morphological operations. Firstly, We binarize JmnM with 1-255 as the threshold, respectively. 
And the 255 binarized images are denoted as $f_{p}^{t h}(\mathrm{~m}, \mathrm{n}),(\mathrm{th}=1,2, \ldots, 255)$, corresponding to 255 marker functions. Referring to (8), maker function can be obtained by performing morphology operation.

$$
\begin{gathered}
f_{\text {Mar }}^{\text {th }}(m, n)=f_{\text {ext }}^{t h}(m, n) \cup f_{\text {int }}^{t h}(m, n) \\
\text { where } \quad f_{\text {ext }}^{\text {th }}(m, n)=\delta_{B_{2}}\left(\delta_{B_{1}}\left(f_{p}^{t h}(m, n)\right)\right)-\varepsilon_{B_{2}}\left(\delta_{B_{1}}\left(f_{p}^{t h}(m, n)\right)\right) \\
\text { and } f_{\text {int }}^{t h}=\varepsilon_{B_{1}}\left(f_{p}^{t h}(m, n)\right)
\end{gathered}
$$

where $f_{p}^{t h}$ represents the marker function; $f_{e x}^{t h}$ and $f_{i n}^{t h}$ represent the external and internal markers, respectively. and $\delta$ and $\varepsilon$ are morphological dilation and erosion, respectively; $B_{1}$ and $B_{2}$ are two structure elements with 15 pixels radius disk and pixel square, respectively.

\section{Segmentation function}

In the paper [26], we discussed and proved that the segmentation function plays a great role in whether we can obtain accurate markers, and has a great contribution to obtain good segmentation results. Therefore, to get more precise segmentation result, we evaluate the existing segmentation methods and proposed an optimized method to obtain the segmentation function.

(1)MS: Let $u_{e}: R^{+} \times R^{2} \rightarrow \mathrm{R}$ be an implicit representation of $\mathrm{C}$ such that $\mathrm{C}(\mathrm{t})=(\mathrm{x}, \mathrm{y}) ; \mathrm{u}(\mathrm{t},(\mathrm{x}, \mathrm{y}))=0$. MS uses a combination of binary morphological operators whose infinitesimal behavior is equivalent to the flow expressed by the active contour PDE(9). Therefore, the curve is given as the zero level set of a binary piecewise constant function $\mathrm{u}: R^{2} \rightarrow\{0,1\}$. We take $\mathrm{u}(\mathrm{x})=1$ for every point $\mathrm{x}$ inside the curve, and $\mathrm{u}(\mathrm{x})=0$ for every point $\mathrm{x}$ outside the curve. The morphological operators will act on $\mathrm{u}$ and will implicitly evolve the curve.

$$
\frac{\partial u}{\partial t}=g(I)|\nabla u|\left(\operatorname{div}\left(\frac{\nabla u}{|\nabla u|}\right)+v\right)+\nabla g(I) \nabla u
$$

where $\mathrm{v} \in \mathrm{R}$ is the balloon force parameter and $\mathrm{g}(\mathrm{I})$ selects which regions of I attract the curve. In the MS model, we used two common morphological operators, which are erosion and dilation. The dilation of a function is defined as:

$$
\left(D_{h} u\right)(\mathrm{x})=\sup _{y \in h B} u(\mathrm{x}-\mathrm{y})
$$

The erosion is defined as

$$
\left(E_{h} u\right)(\mathrm{x})=\inf _{y \in h B} u(\mathrm{x}-\mathrm{y})
$$

The balloon force PDE can be expressed as

$$
\frac{\partial u_{\text {ball }}}{\partial t}=g(I) \bullet V \bullet\left|\nabla u_{\text {ball }}\right|
$$


Given that the snake evolution at iteration $\mathrm{n}, u^{n}: R^{2} \rightarrow\{0,1\}$, it can be solved using the following morphological approach:

$$
u^{n+1}\left(x_{i}\right)=\left\{\begin{array}{cc}
\left(D_{d} u^{n}\right)\left(x_{i}\right) & \text { if } g(I)\left(x_{i}\right)>\theta \text { and } v>0 \\
\left(E_{d} u^{n}\right)\left(x_{i}\right) & \text { if } g(I)\left(x_{i}\right)>\theta \text { and } v<0 \\
u^{n}\left(x_{i}\right) & \text { otherwise }
\end{array}\right.
$$

Where $D_{d}$ and $E_{d}$ are the discrete versions of dilation and erosion. Therefore, the morphological implementation of (13) can be expressed as:

$$
\begin{aligned}
& u^{n+\frac{1}{3}}(x)=\left\{\begin{array}{cc}
\left(D_{d} u^{n}\right)\left(x_{i}\right) & \text { if }|v| g(I)\left(x_{i}\right)>\theta \text { and } v>0 \\
\left(E_{d} u^{n}\right)\left(x_{i}\right) & \text { if }|v| g(I)\left(x_{i}\right)>\theta \text { and } v<0 \\
u^{n}\left(x_{i}\right) & \text { otherwise }
\end{array}\right. \\
& u^{n+\frac{2}{3}}\left(x_{i}\right)=\left\{\begin{array}{cl}
1 & \text { if } \nabla u^{n+\frac{1}{3}} \nabla g(I)\left(x_{i}\right)>0 \\
0 & \text { if } \nabla u^{n+\frac{1}{3}} \nabla g(I)\left(x_{i}\right)<0 \\
u^{n+\frac{1}{3}} & \text { if } \nabla u^{n+\frac{1}{3}} \nabla g(I)\left(x_{i}\right)=0
\end{array}\right. \\
& u^{n+1}\left(x_{i}\right)=\left\{\begin{array}{ccc}
S I_{d} \circ I S_{d} u^{n+\frac{2}{3}}\left(x_{i}\right) & \text { if } & g(I)(x)>0 \\
u^{n+\frac{2}{3}}(x) & \text { otherwise }
\end{array}\right.
\end{aligned}
$$

where $S I_{d}$ and $I S_{d}$ are smoothing operators. In binary images u, $S I_{d}$ works only on white pixels and $I S_{d}$ works only on black pixels. Take $S I_{d}$ for example, for every white pixel $x_{1}$ in a binary image, the $S I_{d}$ operator looks for small ( 3 pixels long) straight lines of white pixels which contain $x_{1}$. This search is done in the four possible orientations corresponding to the four segments in $\mathrm{P}$, where $\mathrm{P}$ is a collection of four discretized segments centered at the origin.

$$
P=\left\{\begin{array}{lll}
\{(0,0), & (1,0), & (-1,0)\}, \\
\{(0,0), & (1,1), & (0,-1)\}, \\
\{(0,0), & (0,1), & (-1,-1)\}, \\
\{(0,0), & (1,-1), & (-1,1)\},
\end{array}\right\}
$$

If no straight line exists, the pixel is made black (see Figure 4). Sharp edges (Figure 4b and Figure 4d) are detected and removed as those pixels which aren't part of a straight line. The white pixels in smooth edges (Figure 4a and Figure 4c) remain unchanged.

If no straight line exists, the pixel is made black (see Figure 4). Sharp edges (Figure $4 \mathrm{~b}$ and $4 \mathrm{~d}$ ) are detected and removed as those pixels which aren't part of a straight line. The white pixels in smooth edges (Figure 4a and Figure 4c) remain unchanged.

(2)AMS: AMS is a optimized model based on MS model, by applying some adjustments on choosing appropriate parameters.

In the AMS model, different shapes and nature of tumors are considered. We use the geometric center of the manually acquired RROI as initial point, and adjust 
the radius and iterations of the circle level set in real time according to the aspect ratio of the tumor, Whereas in MS model, these parameters are fixed. Table 1 lists the relevant adjustable parameters of MS and AMS.

Finally, we can find the minimum boundary fsmth $(m, n)$, referring to (18). Then, we obtained the maker of MW, , by performing closing operation with 25 pixels radius disk after binarization.

$$
f_{s m}^{t h}(m, n)=f_{\text {seg }}(m, n) \cap f_{\text {mar }}^{t h}(m, n)
$$

Final contour acquisition

Firstly, we get 255 candidate contours by putting $f_{l a b e l}^{t h}(\mathrm{~m}, \mathrm{n})$ as the input of MW,referring to (19).

$$
f_{\mathrm{MW}}^{t h}(m, n)=\mathrm{MW}\left(f_{\text {label }}^{t h}(m, n)\right)
$$

Secondly, We take the contour corresponding to the largest average radial derivative (ARD) as the final one, similar to the paper [26]. In order to improve the efficiency of the algorithm and ensure that the selected boundary line is close to the ideal boundary line, we directly take the candidate boundary corresponding to threshold 96 as the final contour line, thus avoiding the calculation of ARD for 255 candidate boundaries in an image. (More details, please refer to paper [26]). Secondly, We take the contour corresponding to the largest average radial derivative (ARD) as the final one, similar to the paper[26]. In order to improve the efficiency of the algorithm and ensure that the selected boundary line is close to the ideal boundary line, we directly take the candidate boundary corresponding to threshold 96 as the final contour line, thus avoiding the calculation of ARD for 255 candidate boundaries in an image. (More details, please refer to paper [26].)

\section{Evaluation Metrics}

We used both area and contour error metrics, which include the accuracy (Acc), the true positive ratio (TPR), false positive ratio (FPR), Jaccard index (JI), Dice's coefficient (DSC), area error ratio (AER), Hausdorff error(HE), and mean absolute error (MAE) to do the evaluation. The calculation formulas of all indicators are listed as below.

$$
\begin{aligned}
& \mathrm{Acc}=\frac{\left(A_{G} \cap A_{S}\right) \cup\left(\mathrm{A}-\mathrm{A}_{\mathrm{G}} \cup \mathrm{A}_{\mathrm{S}}\right)}{\mathrm{A}} \\
& \mathrm{TPR}=\frac{\left|A_{G} \cap A_{S}\right|}{\left|A_{G}\right|} \\
& \mathrm{FPR}=\frac{\left|A_{G} \cup A_{S}-A_{G}\right|}{\left|A_{G}\right|}
\end{aligned}
$$




$$
\begin{aligned}
& \mathrm{JI}=\frac{\left|A_{G} \cap A_{S}\right|}{\left|A_{G} \cup A_{S}\right|} \\
& \mathrm{DSC}=\frac{2\left|A_{G} \cap A_{S}\right|}{\left|A_{G}\right|+\left|A_{S}\right|} \\
& \mathrm{AER}=\frac{\left|A_{G} \cup A_{S}\right|-\left|A_{G} \cap A_{S}\right|}{\left|A_{G}\right|} \\
& \operatorname{HE}\left(C_{G}, C_{S}\right)=\max _{2}\left\{\max _{x \in C_{G}}\left\{d\left(x, C_{S}\right)\right\}\right\}, \max _{x \in C_{S}}\left\{d\left(y, C_{G}\right)\right\} \\
& \operatorname{where,}(z, C)=\min _{k \in C}\{\|z-k\|\} \\
& \operatorname{MAE}\left(\mathrm{C}_{S}, \mathrm{C}_{G}\right)=1 / 2\left(\sum_{x \in C_{S}} \frac{d\left(x, C_{G}\right)}{n_{G}}+\sum_{\mathrm{y} \in C_{G}} \frac{d\left(y, C_{S}\right)}{n_{S}}\right)
\end{aligned}
$$

where $A_{*}$ is the number of pixels exist inside ${ }^{*}, \mathrm{~A}$ is the number of all pixels contained in the image, the subscript $\mathrm{G}$ and $\mathrm{S}$ represent the ground truth and segmentation result, respectively. $\mathrm{C}$ represents the contour of ROI. $\mathrm{z}$ and $\mathrm{k}$ mean the point in the contour.

[12] has mentioned how import these indicators are. The JI value is large, and the AER, HE and MAE value are small, indicating that the performance is quite good. Suppose that JI is small, when AER, HE, and MAE are large, if TPR and FPR are both large, the lesion was overestimated. And if TPR and FPR are both small, the lesion was underestimated.

\section{Experiment details}

In this part, we use Python to implement the algorithm and calculate the evaluation metrics. The best parameter setting of AMSMW method is shown in Table 2. Next, we will introduce the process of the three experiments in detail.

Exploring the effect of preprocessing methods on segmentation results

We explored the effect of preprocessing methods on segmentation results by using four different preprocesing methods, which include SWF, CLAHE+SWF, $\mathrm{CLAHE}+\mathrm{CF}+\mathrm{SWF}$, and $\mathrm{CLAHE}+\mathrm{CF}$, to preprocessl BUS images. In addition, we set up a control group without preprocessing operations, in order to prove whether the preprocessing method has influence on the segmentation result. 
Comparison with other segmentation methods

Firstly, we used the best preprocessing method obtained by the last step to preprocess 500 BUS images. Secondly, we set up a set of comparative experimental groups from the perspective of both the traditional methods and DL.

In terms of traditional segmentation methods, we implemented some related and classical methods, which include LST [5], MS [9], MW [27], FSMW [26], and so on. As for the MS method, we set its initialization position and radius to be the center of the RROI and $70 \%$ of the smallest of the length and width of the RROI, respectively, considering that our dataset is extremely complicated. Moreover, it is necessary to note that we use the same preprocessing method when doing the comparison experiments expect for FSMW. In terms of DL, many excellent image segmentation models have been gradually borrowed, improved and used. In the paper [22], several typical deep learning segmentation models are compared on their testing set. The results show that the RADU-NET model performs best. In order to make an objective comparison between AMSMW and RADU-NET, we did the following two experiments. On one hand, we implemented and trained RADUNET model, using our dataset, which include 1000 BUS images, and performed a quantitative segmentation experiment on our testing set. On the other hand, we performed segmentation experiments on their testing set shared by the paper [22], using AMSMW. In order to facilitate comparison, the indicators used in the paper [22] are used, and the definition of specific indicators will not be repeated here.

Evaluation on the sensitivity in segmenting benign and malignant tumors

It is considered that benign and malignant tumors are very different in size, morphology, margins, and internal state, which may greatly affect the algorithm's performance. If the relationship can be found, it will be of great significance to design a more adaptive BUS segmentation algorithm. Therefore, we conducted an exploratory experiment on the algorithm's sensitivity in segmenting benign and malignant tumors. The specific operation is to firstly group 500 BUS images into 250 benign and 250 malignant groups. and then perform quantitative segmentation experiment.

\section{Abbreviations}

RDAU-NET: Residual-Dilated-Attention-Gate-UNet; CLAHE: contrast limited adaptive histogram equalization; ROI: region of intetrest; SWF: side window filtering; BUS: breast ultrasound; AMSMW: Adaptive morphological snake and marked watershed; DL: deep learning; DM: digital mammography; MS: morpholical snake; FCM: fuzzy cluster method; CNN: Convolutional neural network; SVM: support vector machine; ANN: artificial neural network; DSC: Dice similarity coefficient; WS: Tomography watershed; MW: marked watershed; ADF: anisotropic diffusion filter; CF: curvature filtering; AMS: adaptive morphological snake; LST: level set; RROI: rectangle region of interest; AHE: adaptive histogram equalization; Acc: Accuracy; TPR: true positive ratio; FPR: false positive ratio; JI: Jaccard index; DSC: Dice's coefficient; AER: area error ratio; HE: Hausdorff error; MAE: mean absolute error; 


\section{Declarations}

Ethics approval and consent to participate

This study was approved by the Medical Ethics Committee of the First Hospital of China Medical University and was in accordance with the 1964 Helsinki declaration and its later amendments or comparable ethical standards. All subjects gave written informed consent in accordance with the Declaration of Helsinki.

Consent for publication

Not applicable.

Availability of data and materials

The datasets analyzed during the current study are available from the corresponding author on reasonable request.

Competing interests

The authors declare that they have no competing interests.

Funding

This research is supported in part by the National Natural Science Foundation of China under Grant No. 61702087 and in part by the Fundamental Research Funds for the Central Universities under Grant No. N172008008.

Authors' contributions

XS carried out the algorithm design and implementation, and drafted the manuscript. HM participated in the design of the study, coordination and contributed suggestions to complete the manuscript. RL implemented RDAU-NET.

$\mathrm{HL}$ contributed to discussions. XW participated in discussion, organizing data and writing formulas with latex code. $\mathrm{JH}$ participated in collecting data and delineating tumors. All authors read and approved the final manuscript

Acknowledgements

Not applicable

Authors' information

Author details

${ }^{1}$ the College of Medicine and Biological Information Engineering, Northeastern University, Shenyang, China. ${ }^{2}$ Key Laboratory of Intelligent Computing in Medical Image, Ministry of Education, China. ${ }^{3}$ Department of radiology, Liaoning Cancer Hospital, Shenyang, China.

References

1. Bray, F., Ferlay, J., Soerjomataram, I., Siegel, R.L., Torre, L.A., Jemal, A.: Global cancer statistics 2018: GLOBOCAN estimates of incidence and mortality worldwide for 36 cancers in 185 countries. CA: a cancer journal for clinicians 68(6), 394-424 (2018). doi:10.3322/caac.21492

2. Jemal, A., Bray, F., Center, M.M., Ferlay, J., Ward, E., Forman, D.: Global cancer statistics. CA: a cancer journal for clinicians 61(2), 69-90 (2011)

3. Drukker, K., Giger, M.L., Horsch, K., Kupinski, M.A., Vyborny, C.J., Mendelson, E.B.: Computerized lesion detection on breast ultrasound. Medical Physics 29(7), 1438-1446 (2002). doi:10.1118/1.1485995

4. Geisel, J., Raghu, M., Hooley, R.: The Role of Ultrasound in Breast Cancer Screening: The Case for and Against Ultrasound. Seminars in ultrasound, CT, and MR 39(1), 25-34 (2018). doi:10.1053/j.sult.2017.09.006

5. Sussman, M.: A level set approach for computing solutions to incompressible two-phase flow. Journal of Computational Physics 114(1), 146-159 (1994). doi:10.1006/jcph.1994.1155

6. Adalsteinsson, D., Sethian, J.A.: A fast level set method for propagating interfaces. Journal of computational physics 118(2), 269-277 (1995)

7. Shi, Y., Karl, W.C.: Real-time tracking using level sets. In: 2005 IEEE Computer Society Conference on Computer Vision and Pattern Recognition (CVPR'05), vol. 2, pp. 34-41 (2005). IEEE

8. Gao, L., Liu, X., Chen, W.: Phase- and GVF-based level set segmentation of ultrasonic breast tumors. Journal of Applied Mathematics 2012 (2012). doi:10.1155/2012/810805

9. Álvarez, L., Baumela, L., Henríquez, P., Márquez-Neila, P.: Morphological snakes. Proceedings of the IEEE Computer Society Conference on Computer Vision and Pattern Recognition, 2197-2202 (2010). doi:10.1109/CVPR.2010.5539900

10. Huang, Y.L., Jiang, Y.R., Chen, D.R., Moon, W.K.: Watershed segmentation for breast tumor in 2-D sonography. International Journal of Computer Assisted Radiology and Surgery 1(SUPPL. 7), 63-65 (2006) doi:10.1007/s11548-006-0044-6

11. Gómez, W., Infantosi, A.F.C., Leija, L., Pereira, W.C.A.: Active contours without edges applied to breast lesions on ultrasound. IFMBE Proceedings 29(2), 292-295 (2010). doi:10.1007/978-3-642-13039-7-73

12. Xian, M., Zhang, Y., Cheng, H.D.: Fully automatic segmentation of breast ultrasound images based on breast characteristics in space and frequency domains. Pattern Recognition 48(2), 485-497 (2015) doi:10.1016/j.patcog.2014.07.026

13. Chiang, H.H., Cheng, J.Z., Hung, P.K., Liu, C.Y., Chung, C.H., Chen, C.M.: Cell-based graph cut for segmentation of 2D/3D sonographic breast images. 2010 7th IEEE International Symposium on Biomedical Imaging: From Nano to Macro, ISBI 2010 - Proceedings, 177-180 (2010). doi:10.1109/ISBI.2010.5490384

14. Boukerroui, D., Basset, O., Guérin, N., Baskurt, A.: Multiresolution texture based adaptive clustering algorithm fo breast lesion segmentation. European Journal of Ultrasound 8(2), 135-144 (1998). doi:10.1016/S0929-8266(98)00062-7

15. Zhao, F., Jiao, L., Liu, H.: Kernel generalized fuzzy c-means clustering with spatial information for image segmentation. Digital Signal Processing: A Review Journal 23(1), 184-199 (2013). doi:10.1016/j.dsp.2012.09.016 
16. Lo, C., Shen, Y.W., Huang, C.S., Chang, R.F.: Computer-aided multiview tumor detection for automated whole breast ultrasound. Ultrasonic Imaging 36(1), 3-17 (2014). doi:10.1177/0161734613507240

17. Moon, W.K., Lo, C.M., Chen, R.T., Shen, Y.W., Chang, J.M., Huang, C.S., Chen, J.H., Hsu, W.W., Chang R.F.: Tumor detection in automated breast ultrasound images using quantitative tissue clustering. Medical Physics 41(4) (2014). doi:10.1118/1.4869264

18. Liu, B., Cheng, H.D., Huang, J., Tian, J., Tang, X., Liu, J.: Fully automatic and segmentation-robust classification of breast tumors based on local texture analysis of ultrasound images. Pattern Recognition 43(1), 280-298 (2010). doi:10.1016/j.patcog.2009.06.002

19. Huang, S.F., Chen, Y.C., Woo, K.M.: Neural network analysis applied to tumor segmentation on 3D breast ultrasound images. 2008 5th IEEE International Symposium on Biomedical Imaging: From Nano to Macro, Proceedings, ISBI i, 1303-1306 (2008). doi:10.1109/ISBI.2008.4541243

20. Xu, Y., Wang, Y., Yuan, J., Cheng, Q., Wang, X., Carson, P.L.: Medical breast ultrasound image segmentation by machine learning. Ultrasonics 91(March 2018), 1-9 (2019). doi:10.1016/j.ultras.2018.07.006

21. Ronneberger, O., Fischer, P., Brox, T.: U-net: Convolutional networks for biomedical image segmentation. In: International Conference on Medical Image Computing and Computer-assisted Intervention, pp. 234-241 (2015). Springer

22. Zhuang, Z., Li, N., Joseph Raj, A.N., Mahesh, V.G., Qiu, S.: An rdau-net model for lesion segmentation in breast ultrasound images. PloS one 14(8), 0221535 (2019)

23. Vakanski, A., Xian, M., Freer, P.E.: Attention-enriched deep learning model for breast tumor segmentation in ultrasound images. Ultrasound in Medicine \& Biology 46(10), 2819-2833 (2020)

24. Gómez, W., Leija, L., Pereira, W.C.A., Infantosi, A.F.C.: Morphological operators on the segmentation of breast ultrasound images. 2009 Pan American Health Care Exchanges - PAHCE 2009, 67-71 (2009). doi:10.1109/PAHCE.2009.5158367

25. Gómez, W., Leija, L., Alvarenga, A.V., Infantosi, A.F.C., Pereira, W.C.A.: Computerized lesion segmentation of breast ultrasound based on marker-controlled watershed transformation. Medical Physics 37, 82-95 (2010). doi:10.1118/1.3265959

26. Shen, X., Liu, J., Li, H., Sun, H., Ma, H.: A novel lesion segmentation method based on breast ultrasound images. ACM International Conference Proceeding Series, 32-38 (2019). doi:10.1145/3366174.3366176

27. Lewis, S.H., Dong, A.: Detection of breast tumor candidates using marker-controlled watershed segmentation and morphological analysis. In: 2012 IEEE Southwest Symposium on Image Analysis and Interpretation, pp. 1-4 (2012). IEEE

28. Yin, H., Gong, Y., Qiu, G.: Side window filtering. In: Proceedings of the IEEE Conference on Computer Vision and Pattern Recognition, pp. 8758-8766 (2019)

Figures

Figure 1 Flowchart of the proposed method

Figure 2 Definition of side window. $r$ is the radius of the window. (a) side window in continuous case. (b) The left (L) and right (R) side window. (c) The up (U) and down (D) side window. (d) The northeast (NE), northwest (NW), southeast (SE) and southwest (SW) side window.

Figure 3 The process of obtaining ROI(region of interest). (a) original image with RROI(rectangle region of interest) draw by hands; (b) a constrained Gaussian function centered at the geometric center of RROI; (c) resultant image after multiplying (b) and (d); (d) negative of $I_{C F}$; (e) the union of five constrained Gaussian functions; (f)resultant image, which is denoted as J, after multiplying (e) and (d).

Figure 4 Some example of the effect of the $S I_{d}$ and $I d_{S}$ operator. It keeps unchanged where a straight line(marked in red) is found, as shown in (a) and (c). However, when the center point isn't in any straight line, it will be changed as shown in (b) and (d) 
Figure 5 Some examples of the effect of different preprocess methods. From the first row to the last row are the result of CCS(contrast limited adaptive histogram equalization+curvature filter+side window filter), CC(contrast limited adaptive histogram equalization+curvature filte), $\mathrm{CS}$ (contrast limited adaptive histogram equalization + side window filter) and SWF(side window filter) respectively.

Figure 6 From up to down are the qualitative result of MW(markerd watershed), LST(level set), MS(morphological snake), AMS(adaptive morphological snake), MSMW(morphological snake and marked watershed), AMSMW(Adaptive morphological snake and marked watershed), LSMW(level set and marked watershed), FSMW and GT(ground truth)

Figure 7 The qualitative segmentation result. The first row is GT and the second row is the qualitative result of AMSMW on the shared database. And from left to the right are image(a), image(b), image(c), image(d), image(e), image(f), image(g), image(h), image(i), image(j), image $(k)$ and image $(\mathrm{I})$. All of them can be found in the figure11,figure12 and figure 13 of Zhuang's paper ([22]) accordingly.

Figure 8 From up to down are the qualitative result of RADU-NET and GT, respectively.

Table 1 The adjustable parameters of MS(morphological snake) and AMS(adaptive morphological snake).

\begin{tabular}{|c|c|c|c|}
\hline method & initial point & Radius & iterations \\
\hline MS & $\begin{array}{l}\text { A fixed point (default } \\
\text { to be the center of the } \\
\text { image) }\end{array}$ & $\begin{array}{l}\text { A fixed value (default } \\
\text { to be } 75 \% \text { of the } \\
\text { smallest image } \\
\text { dimension) }\end{array}$ & a fixed value \\
\hline AMS & $\begin{array}{l}\text { the geometric center of } \\
\text { RROI }\end{array}$ & $\begin{array}{c}\text { Real-time adjusted } \\
\text { value }\end{array}$ & $\begin{array}{c}\text { Real-time adjusted } \\
\text { value }\end{array}$ \\
\hline
\end{tabular}

Table 2 The optimal parameters value of the AMSMW method.

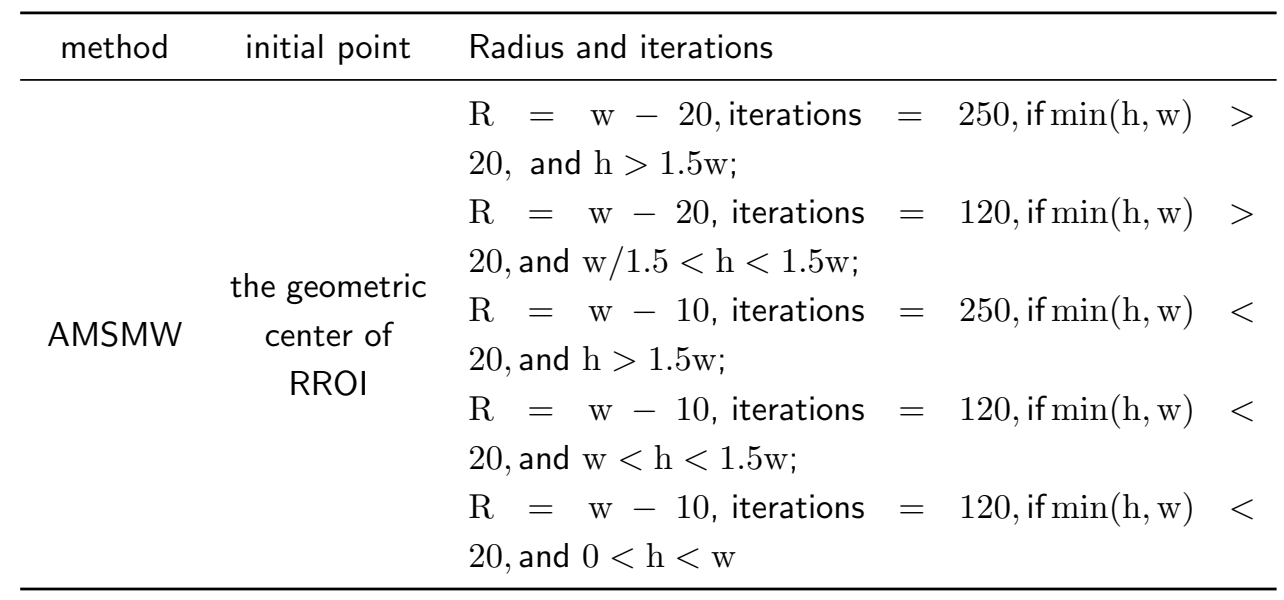


Table 3 the quantitative result of exploring the effect of preprocessing methods on segmentation results. (CC:CLAHE+CF; CCS: $C L A H E+C F+S W F$; CS: CLAHE+SWF* Values are the average ( \pm standard deviation) for 500 images)

\begin{tabular}{cccccc}
\hline & None & SWF & CC & CCS & CS \\
\hline Acc(\%) & 94.48 & 95.35 & 96.25 & 96.19 & $\mathbf{9 6 . 5 9}$ \\
& $( \pm 0.04)$ & $( \pm 0.04)$ & $( \pm 0.03)$ & $( \pm 0.03)$ & $( \pm 0.03)$ \\
TPR(\%) & 91.323 & $\mathbf{9 2 . 5 7}$ & 0.9004 & 89.11 & 83.29 \\
& $( \pm 0.12)$ & $( \pm 0.08)$ & $( \pm 0.09)$ & $( \pm 0.09)$ & $( \pm 0.09)$ \\
FPR(\%) & 59.57 & 53.14 & 41.90 & 41.92 & $\mathbf{1 . 9 4}$ \\
& $( \pm 0.19)$ & $( \pm 0.17)$ & $( \pm 0.18)$ & $( \pm 0.18)$ & $( \pm 0.17)$ \\
DSC(\%) & 73.25 & 76.23 & 78.39 & 77.86 & $\mathbf{7 8 . 4 0}$ \\
& $( \pm 0.08)$ & $( \pm 0.08)$ & $( \pm 0.08)$ & $( \pm 0.08)$ & $( \pm 0.08)$ \\
JI(\%) & 58.36 & 62.25 & 65.17 & 64.48 & $\mathbf{6 5 . 3 4}$ \\
& $( \pm 0.09)$ & $( \pm 0.10)$ & $( \pm 0.11)$ & $( \pm 0.11)$ & $( \pm 0.11)$ \\
AER(\%) & 68.25 & 60.56 & 51.86 & 52.81 & $\mathbf{4 6 . 4 5}$ \\
& $( \pm 0.26)$ & $( \pm 0.30)$ & $( \pm 0.30)$ & $( \pm 0.29)$ & $( \pm 0.26)$ \\
HE & 66.37 & 60.90 & 54.36 & 54.65 & $\mathbf{5 1 . 3 3}$ \\
& $( \pm 32.01)$ & $( \pm 31.27)$ & $( \pm 28.94)$ & $( \pm 28.61)$ & $( \pm 29.54)$ \\
MAE & 26.12 & 22.36 & 18.77 & 19.15 & $\mathbf{1 7 . 7 0}$ \\
& $( \pm 11.12)$ & $( \pm 9.20)$ & $( \pm 7.30)$ & $( \pm 7.26)$ & $( \pm 7.65)$ \\
\hline
\end{tabular}

Table 4 the quantitative result of different segmentation methods.

\begin{tabular}{ccccccccc}
\hline & ACC & TPR & FPR & DSC & Jl & AER & HE & MAE \\
& $(\%)$ & $(\%)$ & $(\%)$ & $(\%)$ & $(\%)$ & $(\%)$ & & \\
\hline MW & 94.37 & $\mathbf{9 7 . 6 4}$ & 79.17 & 71.80 & 56.68 & 81.53 & 69.21 & 27.88 \\
& $( \pm 0.03)$ & $( \pm 0.02)$ & $( \pm 0.05)$ & $( \pm 0.08)$ & $( \pm 0.10)$ & $( \pm 0.41)$ & $( \pm 26.97)$ & $( \pm 8.81)$ \\
LST & 94.69 & 95.56 & 5.24 & 69.58 & 54.83 & 98.11 & 72.54 & 28.09 \\
& $( \pm 0.03)$ & $( \pm 0.05)$ & $( \pm 0.03)$ & $( \pm 0.13)$ & $( \pm 0.14)$ & $( \pm 0.78)$ & $( \pm 26.35)$ & $( \pm 9.06)$ \\
MS & 95.76 & 63.05 & 11.93 & 71.57 & 56.72 & 48.88 & 69.91 & 22.68 \\
& $( \pm 0.04)$ & $( \pm 0.13)$ & $( \pm 0.16)$ & $( \pm 0.10)$ & $( \pm 0.12)$ & $( \pm 0.17)$ & $( \pm 50.21)$ & $( \pm 13.67)$ \\
AMS & 94.38 & 81.70 & 45.32 & 72.69 & 58.01 & 63.62 & 72.72 & 24.94 \\
& $( \pm 0.05)$ & $( \pm 0.15)$ & $( \pm 0.28)$ & $( \pm 0.09)$ & $( \pm 0.11)$ & $( \pm 0.32)$ & $( \pm 44.87)$ & $( \pm 14.53)$ \\
MSMW & 95.92 & 67.65 & 15.40 & 73.57 & 59.14 & 47.74 & 67.00 & 21.75 \\
& $( \pm 0.03)$ & $( \pm 0.04)$ & $( \pm 0.11)$ & $( \pm 0.12)$ & $( \pm 0.14)$ & $( \pm 0.73)$ & $( \pm 25.24)$ & $( \pm 7.13)$ \\
AMSMW & $\mathbf{9 6 . 5 9}$ & 83.29 & $\mathbf{1 . 9 4}$ & $\mathbf{7 8 . 4 0}$ & $\mathbf{6 5 . 3 4}$ & $\mathbf{4 6 . 4 5}$ & $\mathbf{5 1 . 3 3}$ & $\mathbf{1 7 . 7 0}$ \\
& $( \pm 0.02)$ & $( \pm 0.11)$ & $( \pm 0.21)$ & $( \pm 0.09)$ & $( \pm 0.11)$ & $( \pm 0.22)$ & $( \pm 28.66)$ & $( \pm 7.37)$ \\
LSMW & 96.25 & 91.58 & 50.52 & 77.47 & 64.18 & 58.94 & 55.85 & 19.50 \\
& $( \pm 0.03)$ & $( \pm 0.06)$ & $( \pm 0.20)$ & $( \pm 0.09)$ & $( \pm 0.12)$ & $( \pm 0.45)$ & $( \pm 27.01)$ & $( \pm 7.36)$ \\
FSMW & 96.19 & 92.88 & 52.19 & 77.53 & 64.27 & 59.30 & 56.08 & 19.50 \\
& $( \pm 0.03)$ & $( \pm 0.05)$ & $( \pm 0.14)$ & $( \pm 0.09)$ & $( \pm 0.12)$ & $( \pm 0.45)$ & $( \pm 28.61)$ & $( \pm 7.21)$ \\
\hline
\end{tabular}

Table 5 the quantitative result of AMSMW on the database shared by paper [22]

\begin{tabular}{ccccccccc}
\hline & $\begin{array}{c}\text { loss } \\
(\%)\end{array}$ & $\begin{array}{c}\text { Acc } \\
(\%)\end{array}$ & $\begin{array}{c}\text { DC } \\
(\%)\end{array}$ & $\begin{array}{c}\text { Sen } \\
(\%)\end{array}$ & $\begin{array}{c}\text { Sp } \\
(\%)\end{array}$ & $\begin{array}{c}\text { F1 } \\
(\%)\end{array}$ & $\begin{array}{c}\text { Pc } \\
(\%)\end{array}$ & $\begin{array}{c}\text { M-IOU } \\
(\%)\end{array}$ \\
\hline UNet & 17.95 & 97.57 & 82.04 & 84.66 & 98.91 & 82.11 & 81.85 & 79.83 \\
RDAU & 15.30 & 97.91 & 84.69 & 83.19 & $\mathbf{9 9 . 3 4}$ & 84.78 & $\mathbf{8 8 . 5 8}$ & $\mathbf{8 0 . 6 7}$ \\
AMSMW & $\mathbf{1 3 . 7 5}$ & $\mathbf{9 7 . 9 6}$ & $\mathbf{8 6 . 2 5}$ & $\mathbf{8 8 . 7 9}$ & 98.32 & $\mathbf{8 6 . 2 5}$ & 86.26 & 76.73 \\
\hline
\end{tabular}

Table 6 the quantitative result of AMSMW on our database.

\begin{tabular}{ccccccccc}
\hline & loss & Acc & DC & Sen & Sp & F1 & Pc & M-IOU \\
$(\%)$ & $(\%)$ & $(\%)$ & $(\%)$ & $(\%)$ & $(\%)$ & $(\%)$ & $(\%)$ \\
\hline RDAU & 36.73 & 92.65 & 63.27 & $\mathbf{9 2 . 3 5}$ & 92.62 & 63.27 & 52.43 & 49.45 \\
AMSMW & $\mathbf{2 1 . 6 0}$ & $\mathbf{9 6 . 5 9}$ & $\mathbf{7 8 . 4 0}$ & 83.29 & $\mathbf{9 7 . 2 1}$ & $\mathbf{7 8 . 4 0}$ & $\mathbf{7 5 . 4 8}$ & $\mathbf{6 5 . 3 4}$ \\
\hline
\end{tabular}


Table 7 the quantitative result of study on algorithm's sensitivity in segmenting benign and malignant tumors. (B: Benign tunor; $\mathrm{M}$ : malignant tumor)

\begin{tabular}{ccccccccc}
\hline & ACC & TPR & FPR & DSC & JI & AER & HE & MAE \\
& $(\%)$ & $(\%)$ & $(\%)$ & $(\%)$ & $(\%)$ & $(\%)$ & & \\
\hline B & $\mathbf{9 7 . 4 6}$ & 79.54 & $\mathbf{1 . 9 4}$ & 76.24 & 62.63 & 50.03 & $\mathbf{4 2 . 1 5}$ & $\mathbf{1 5 . 7 1}$ \\
M & 95.71 & $\mathbf{8 7 . 0 3}$ & 3.65 & $\mathbf{8 0 . 5 5}$ & $\mathbf{6 8 . 0 5}$ & $\mathbf{4 2 . 8 7}$ & 60.51 & 19.68 \\
\hline
\end{tabular}

